\title{
Effect Analysis of Virtual-reality Vestibular Rehabilitation based on Eye-tracking
}

\author{
Sungjin Lee ${ }^{1}$, Min Hong ${ }^{2}$, Sungyeup $\mathrm{Kim}^{3}$, and Seong Jun Choi ${ }^{{ }^{*}}$ \\ ${ }^{1}$ Department of Computer Science, Soonchunhyang University, Asan, South Korea \\ ${ }^{2}$ Department of Computer Software Engineering, Soonchunhyang University, Asan, South Korea \\ ${ }^{3}$ Department of ICT Convergence Rehabilitation Engineering, Soonchunhyang University, \\ Asan, South Korea \\ ${ }^{4}$ Department of Otorhinolaryngology-Head and Neck Surgery, College of Medicine, Soonchunhyang \\ University, Cheonan Hospital, Cheonan, South Korea \\ [e-mail: fijianwa@gmail.com,mhong@sch.ac.kr,sykim@sch.ac.kr, and akas9238@hanmail.net] \\ *Corresponding author: Seong Jun Choi
}

\section{Received Septebmer 15, 2019; revised December 5, 2019; accepted January 2, 2019;} published February 29, 2020

\begin{abstract}
Vertigo is one of the most common complaints encountered by physicians and the patients are steadily increasing. These patients are exposed to the risk of secondary accidents such as falls due to vertigo. There are two ways to improve this symptom: medication and rehabilitation. Although temporary symptomatic improvement may be expected in patients treated with medication, vertigo may recur and medication can delay central compensation. In contrast vestibular rehabilitation exploits central mechanisms of neuroplasticity to increase postural stability and enhance visual-vestibular interactions in situations that generate conflicting sensory information. However, vestibular rehabilitation may be compromised by incorrect performance of exercises, and there is a need for active effort and interest from the patient during rehabilitation. To solve these problems, we decided to apply FOVE HMD for eye-tracking and Unity3D to create virtual reality. The proposed eye-tracking based algorithm calculates the concentration of users with eye tracking data and calculates the motion width of the patient with nystagmus, thus the severity of the patient according to the score can be determined. According to our experimental test against healty group and patients group, this result showed the meaningful data to use define the contents result.
\end{abstract}

Keywords: Virtual Reality, Eye-tracking, Vertigo, Vestibular Rehabilitation, FOVE, Unity3D, HMD

A preliminary version of this paper was presented at APIC-IST 2019, and was selected as an outstanding paper. This research was supported by the MSIP(Ministry of Science, ICT and Future Planning), Korea, under the ITRC(Information Technology Research Center) support program (IITP-2018-2014-1-00720) supervised by the IITP(Institute for Information \& communications Technology Promotion)" and this research supported by Soonchunhyang University's research fund. 


\section{Introduction}

Vertigo is one of the most common complaints encountered by physicians. Balance disorders of vestibular origin have a lifetime prevalence of 7.4\% [1], and include benign paroxysmal positional vertigo and Meniere's disease-associated vertigo. Patients with vestibular dysfunction complain of vertigo, difficulty balancing, and falling episodes. They may also report blurred vision on movement, such that head movements are required for balance while walking. In particular, elderly populations show a high incidence of vertigo, and may experience accidents due to the condition; thus, they should be closely monitored. Both medications and vestibular rehabilitation can improve symptoms. Although temporary symptomatic improvement may be expected in patients treated with medication, vertigo may recur and medication can delay central compensation.

In contrast, vestibular rehabilitation exploits central mechanisms of neuroplasticity (adaptation, habituation, and substitution) to increase static and dynamic postural stability and enhance visual-vestibular interactions in situations that generate conflicting sensory information [2-4]. Moreover, vestibular rehabilitation may improve static and dynamic balance and gait, thus reducing symptoms of dizziness and comorbid depression and anxiety; this ultimately increases the self-confidence and quality of life of affected patients [5]. Vestibular rehabilitation is also regarded as a safe and effective treatment modality for patients with vestibular disorders. Previous researches have shown that patients referred to vestibular rehabilitation programs at an early juncture show less disability and better gait performance after vestibular deafferentation [6-8].

However, vestibular rehabilitation may be compromised by incorrect performance of exercises, and there is a need for active effort and interest from the patient during rehabilitation; thus, efficacy varies among patients. To overcome these limitations, VR (Virtual Reality) has recently attracted attention. VR systems enable real-time simulations, have interactive functions and game-like features that promote mechanisms of neuroplasticity (adaptation, habituation, and substitution), and can improve patient motivation during vestibular rehabilitation [9]. HMD (Head Mounted displays) that provide a fully immersive experience are commonly used clinically because of their affordability and portability [10]. Also, HMDs provide high-resolution images that mimic the user's movements, and thus give rise to an immersive experience [11].

Many previous researches have indicated that VR can improve the likelihood of successful clinical outcomes [12-15]. Additionally, VR enables visual-vestibular interactions due to abundant visual stimuli, and provides an optimized environment for rehabilitation [16]. Saprer et al. [17] reported that use of the Wii Fit Plus device during the first 2 weeks after acute vestibular neuritis was more effective in improving balance compared with placebo. However, the efficacy of VR-based vestibular rehabilitation remains controversial [18]. Clinical optimization of immersive wearable devices, such as HMDs, for dizzy patients is still required. Meldrum et al. reported that VR-based balance exercises performed during vestibular rehabilitation were not superior to conventional balance exercises during vestibular rehabilitation, but may constitute a more enjoyable modality for retraining balance after unilateral peripheral vestibular loss. VR-based vestibular rehabilitation outcomes may differ according to patient factors such as concentration levels and task accuracy. Park et al. showed that eye tracking algorithms can enhance vestibular rehabilitation using HMDs.

Here, we developed a VR-based vestibular rehabilitation program with an eye-tracking based algorithm created using the Unity3D engine. The purpose of this paper was to determine the 
utility of eye-tracking in vestibular rehabilitation, and to analyze the correlation between patient condition and VR-vestibular rehabilitation program.

\section{Related Work}

Vestibular rehabilitation training was developed in the 1940s to rehabilitate patients with brain damage and dizziness. After vestibulopathy, we observed that recovery was rapid when head movement was substantial; thus, we designed a rehabilitation method based on this principle [19-20]. In a comparison of vestibular suppressant treatment, general exercise, and vestibular rehabilitation exercise groups, the rehabilitation exercise group experienced the greatest symptom relief; in the other experimental groups, treatment was either ineffective or associated with worse outcomes [21].

The goal of vestibular rehabilitation training is to improve balance ability and vestibular function via adaptation and strengthening. The Cawthorne-Cooksey exercise is a commonly used rehabilitation exercise that is easy to implement and inexpensive. In this research, we used three rehabilitation exercises that can readily be performed in a VR environment. In addition, a vestibular adaptation exercise resulting in persistent and severe dizziness was used to habituate the patients to the sensation of dizziness; it was performed under the guidance of a specialist and patients were continuously monitored. A typical vestibular adaptation exercise is Herdman's exercise [22] and other exercises include the ball exercise and walking in a straight line..

\subsection{Cawthorne - Cooksey Training}
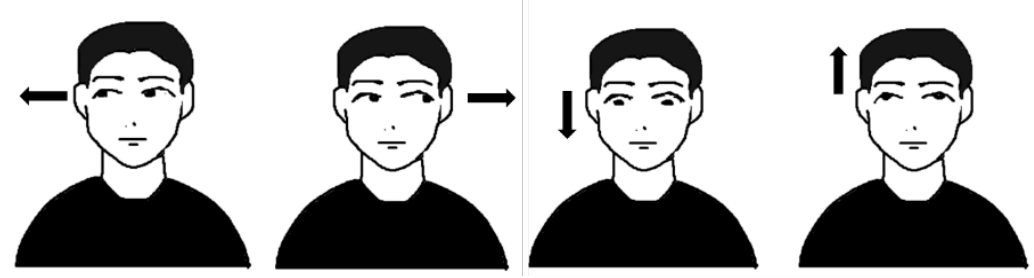

Fig. 1. Cawthorne - Cooksey Training \#1

a) Eye moves left and right

b) Eye moves down and up

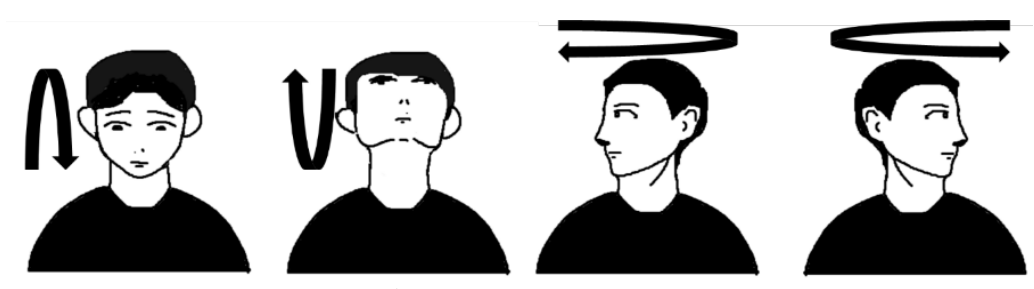

Fig. 2. Cawthorne - Cooksey Training \#2

a) Head moves down and up

b) Head moves left and right rotating head movement 
Cawthorne - Cooksey training is shown in Fig. 1 and Fig. 2. The Cawthorne-Cooksey exercise can be performed in a lying, sitting, or standing position, and during movement; the lying position can be omitted from rehabilitation training. This research excluded difficult exercises for patients with HMD because these should be done in consultation with dizziness specialists.

\subsection{Herdman training}

Fig. 3 shows how to exercise Herdman training.
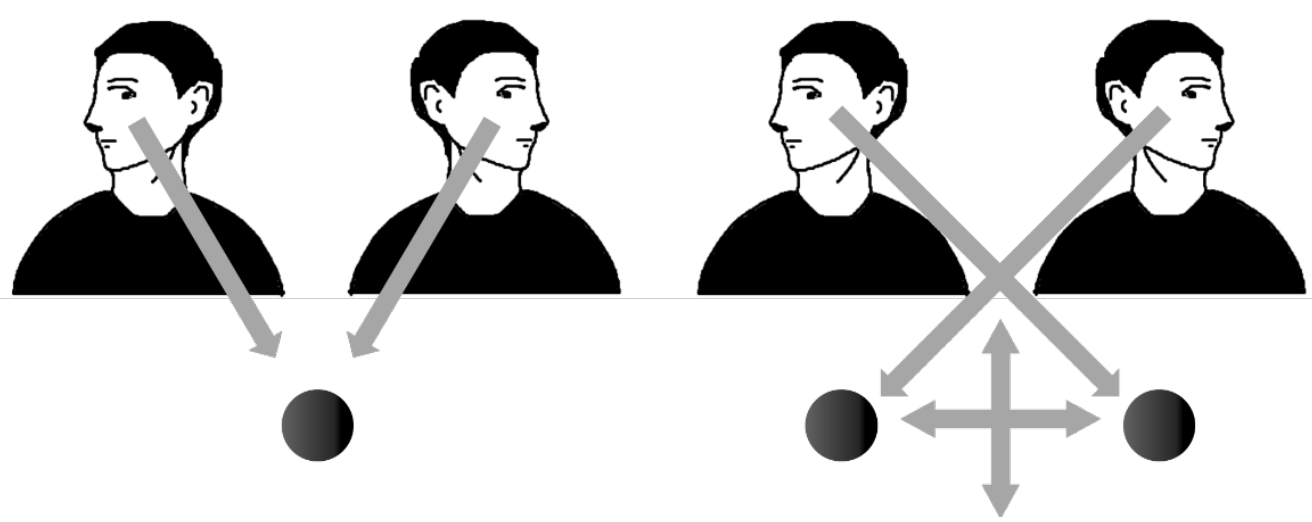

Fig. 3. Herdman training

a) Target should stop, eye should focus the traget when head move left, right, up, and down

b) Target should move to left, right, up and down. Eyes must focus on a target but head should be opposite direction with target.

\subsection{Effect of vestibular rehabilitation training}

In a research by Cohen et al. [23], individualized vestibular rehabilitation training resulted in better outcomes than general training, regardless of age, gender, and training duration. Rhee et al. [24] conducted vestibular rehabilitation training at Dankook University Hospital; patients with bilateral or central lesions showed significant improvements.

\subsection{VR technology}

Using VR technology, virtual environments and objects can be created and interacted with via the visual, auditory, and tactile senses [25]. This approach has fewer spatial and physical constraints than personal computer-based experimental paradigms, thus enhancing immersion [26-27]. We used the Unity physics engine to create the VR content used in this research. Unity3D can be used on multiple platforms and has a highly intuitive graphical user interface [28-29]. In this paper, we used an eye-tracking-enabled FOVE HMD which provides the frame rate was high to prevent dizziness. Table $\mathbf{1}$ shows the specification of recent HMDs. 
Table 1. Specifications of recent HMDs

\begin{tabular}{|c|c|c|c|}
\hline Division & FOVE & $\begin{array}{c}\text { HTC } \\
\text { VIVE }\end{array}$ & Gear VR \\
\hline Platform & PC & PC & $\begin{array}{c}\text { Mobile } \\
\text { (Android) }\end{array}$ \\
\hline FPS & $120 \mathrm{FPS}$ & $90 \mathrm{FPS}$ & $60 \mathrm{FPS}$ \\
\hline Controller & $\mathrm{X}$ & $\bigcirc$ & $\mathrm{X}$ \\
\hline FOV & $100^{\circ}$ & $110^{\circ}$ & $101^{\circ}$ \\
\hline Display & $2560 \times 1440$ & $2160 \times 1200$ & $\begin{array}{c}\text { Depends on } \\
\text { SAMSUNG } \\
\text { smartphone }\end{array}$ \\
\hline
\end{tabular}

The user interface may not work properly when the eyeball is not tracked accurately such that, when the user wears the HMD, the eye is not measured; in this situation, the user interface is operated based on the most recently obtained left and right eye coordinates. The mean values are regarded as the "central coordinates", according to which motion is tracked. For the HMD we tested on i7-3770K CPU with 24.0GB RAM and use NVIDIA GeForce GTX 1060 6GB. To use FOVE HMD software version of FOVE is 0.14.1 and made contents in Unity 2017.1.1f1.

\section{Implementation of vestibular rehabilitation training contents using VR}

\subsection{Subjects}

This research was approved by our Institutional Review Board in by the ethics guidelines established by the Institutional Review Board of Soonchunhyang University Schools of Medicine. Our research included 10 healthy volunteers and 9 patients with unilateral vestibular dysfunction. The healthy volunteers recruited in this research were between 26 to 38 years of age with no reports of middle ear pathology and had never suffered from dizziness, vertigo, and imbalance. The patients in the research were recruited from the 'vestibular clinic' of otorhinolaryngology department of our school hospital. Patients who complained of vertigo or dizziness more than two weeks and showed unilateral vestibular dysfunction using an electro-nystagmogram were enrolled. The inclusion criteria in our research were 1) age between 20 to 70 years, 2) a clinical diagnosis of unilateral vestibular dysfunction, and 3) have complaints including imbalance, and vertigo/dizziness more than 2 weeks. Exclusion criteria included the following: 1) they were unwilling to use the VR-vestibular rehabilitation system, 2 ) age $<19$ years, 3) any central nervous system disorder, 4) vestibular depressant medication, and 5) use of assistive devices for ambulation. All research participant was provided with an information regarding the study.

\subsection{Intervention}

Patients were trained on the VR-based vestibular rehabilitation system for 20-30 min while seated on a chair. All patients completed a single VR-based vestibular rehabilitation exercise. For each of the three exercises (i.e., "games"), participants started on the lowest difficulty 
level; an adaptive algorithm automatically changed the difficulty level. If participants complained of headache or any other symptom related to the training, it was immediately stopped. The healthy subjects were tested to obtain normative game score data. They performed a single exercise, as per the patient group. For all participants, eye movement accuracy and scores were obtained. Fig. 4. shows the algorithm for proposed VR-based vestibular rehabilitation system.

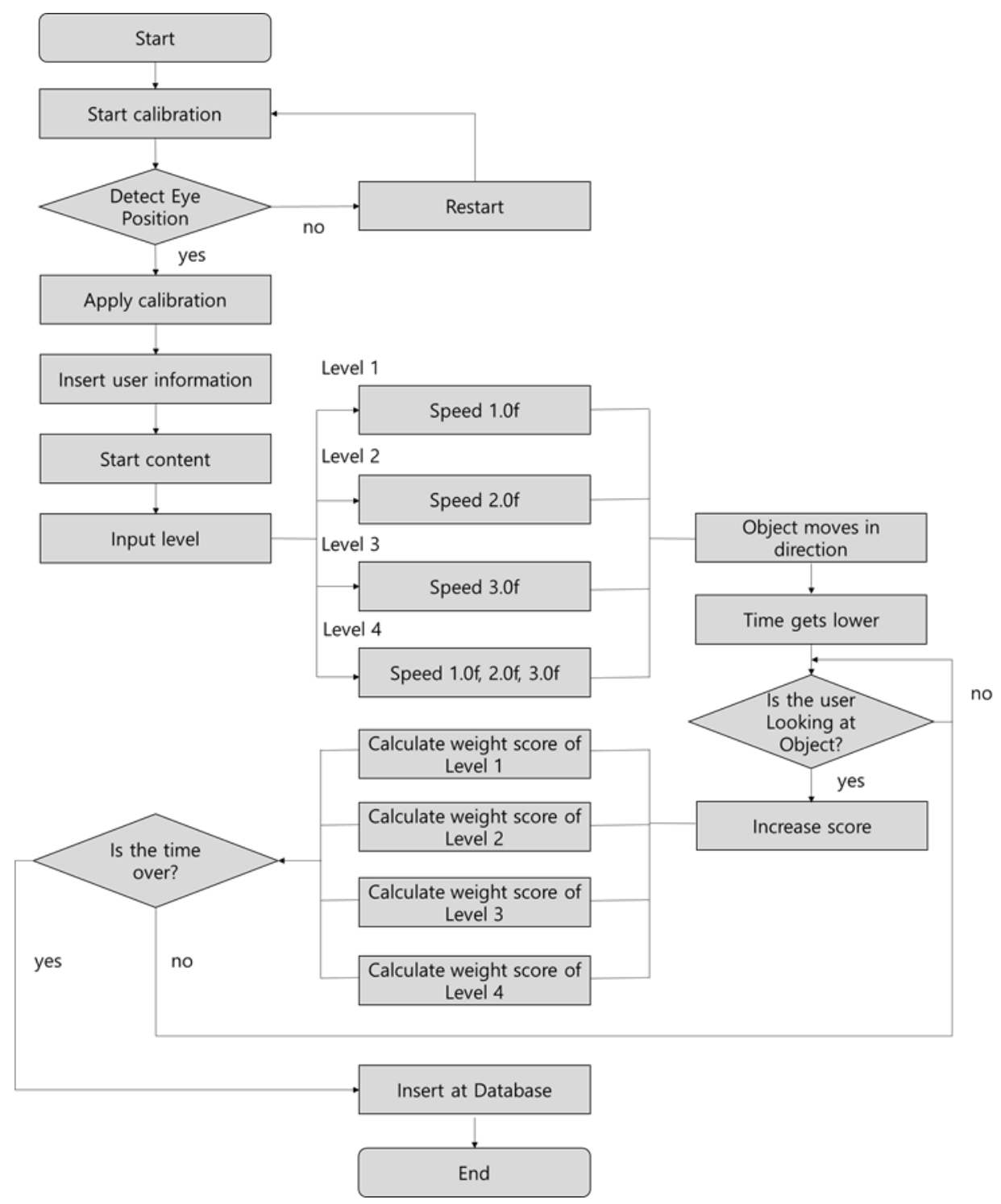

Fig. 4. Flowchart of algorithm for VR-based vestibular rehabilitation system

\subsection{Outcome measures}

During the contents, the sight of user should follow at the movement of target via their eye movements. When the sight of user hit a target, the target changed to a different color and the user gained points; the final score was the outcome measure. 


$$
\text { Score }=\frac{\text { Successful hit time }}{\text { Play time }} * 100 \quad \text { Weight Score }=\text { Score } * \text { Speed of Object }
$$

For the eye movement we record the eye-tracking data, vector value of eye, gaze value of eye and position of eye. The data saved for $70 \mathrm{hz}$ and it means that record the data 70 data in 1 second and we made a graph for it. The graph's number of x-axis and y-axis doesn't have proper unit like $\mathrm{mm}$ or $\mathrm{nm}$. Because we implemented the contents in Unity3D and it doesn't have exact unit.

\subsection{VR-vestibular rehabilitation system based on Eye-tracking}

\subsubsection{Eye movement exercise: the proposed vestibular rehabilitation content I}

During the proposed vestibular rehabilitation content I, the head should be fixed and a target should be followed with the eye. The target moved leftward and rightward. The FOVE HMD can detect the eye's original position, the eye vector, the gaze direction, and eye blinks, as well as head position and movement. A circle ("aim") was generated to represent the movement of the eye: when the aim touched a target, the user received points and scores were weighted by level (100 points on level 1 difficulty was thus not the same as 100 points on level 3, for example). When the user moved their head in any direction, a warning message was displayed in the middle of the field of view. Fig. 5 explains the training process of proposed vestibular rehabilitation content I for Cawthorne-Cooksey training

Experiment A : Level 1(Speed of object is 1) smoothly moving for $40 \mathrm{sec}$

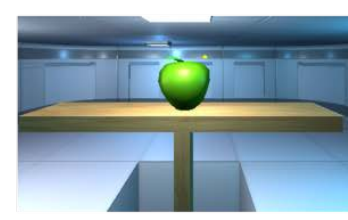

Default Position

Fail to hit a target by user sight

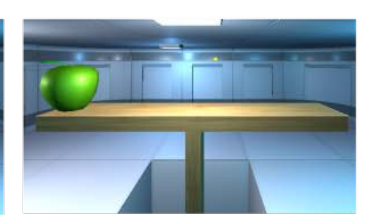

Left Position

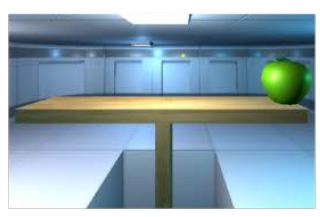

Right Position

Fail to hit a target by user sight
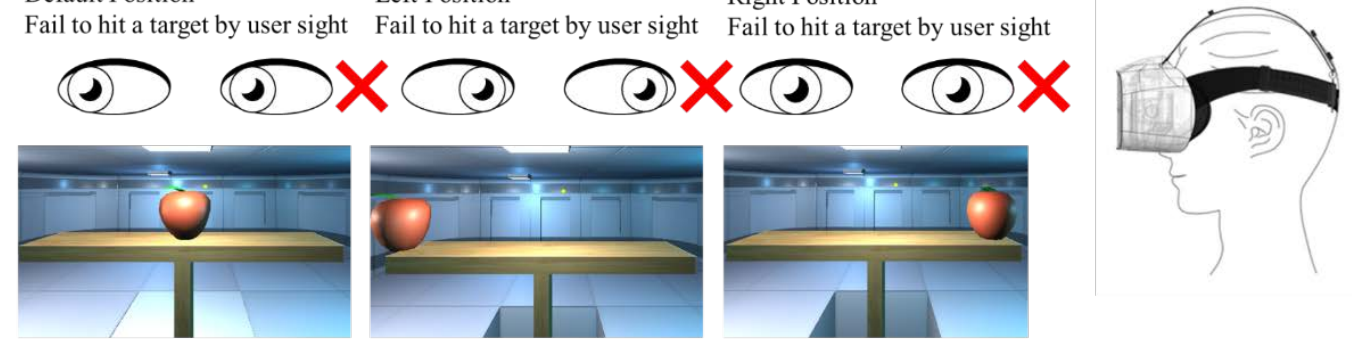

Default Position

Left Position

Right Position

Success to hit a target by user sight Success to hit a target by user sight Success to hit a target by user sight

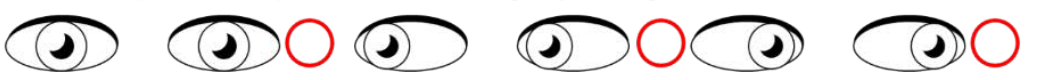

Experiment B : Level 2(Speed of object is 2) smoothly moving for $40 \mathrm{sec}$

Experiment C : Level 3(Speed of object is 3) smoothly moving for $40 \mathrm{sec}$

Fig. 5. The proposed vestibular rehabilitation content I for Cawthorne-Cooksey training 


\subsubsection{Eye movement exercise: the proposed vestibular rehabilitation content II}

The proposed vestibular rehabilitation content II also required the head to be fixed and the target to be followed with the eye; the only difference from content I was that the target moved upward and downward. Fig. 6 shows the training process of proposed vestibular rehabilitation content II for Cawthorne-Cooksey training.

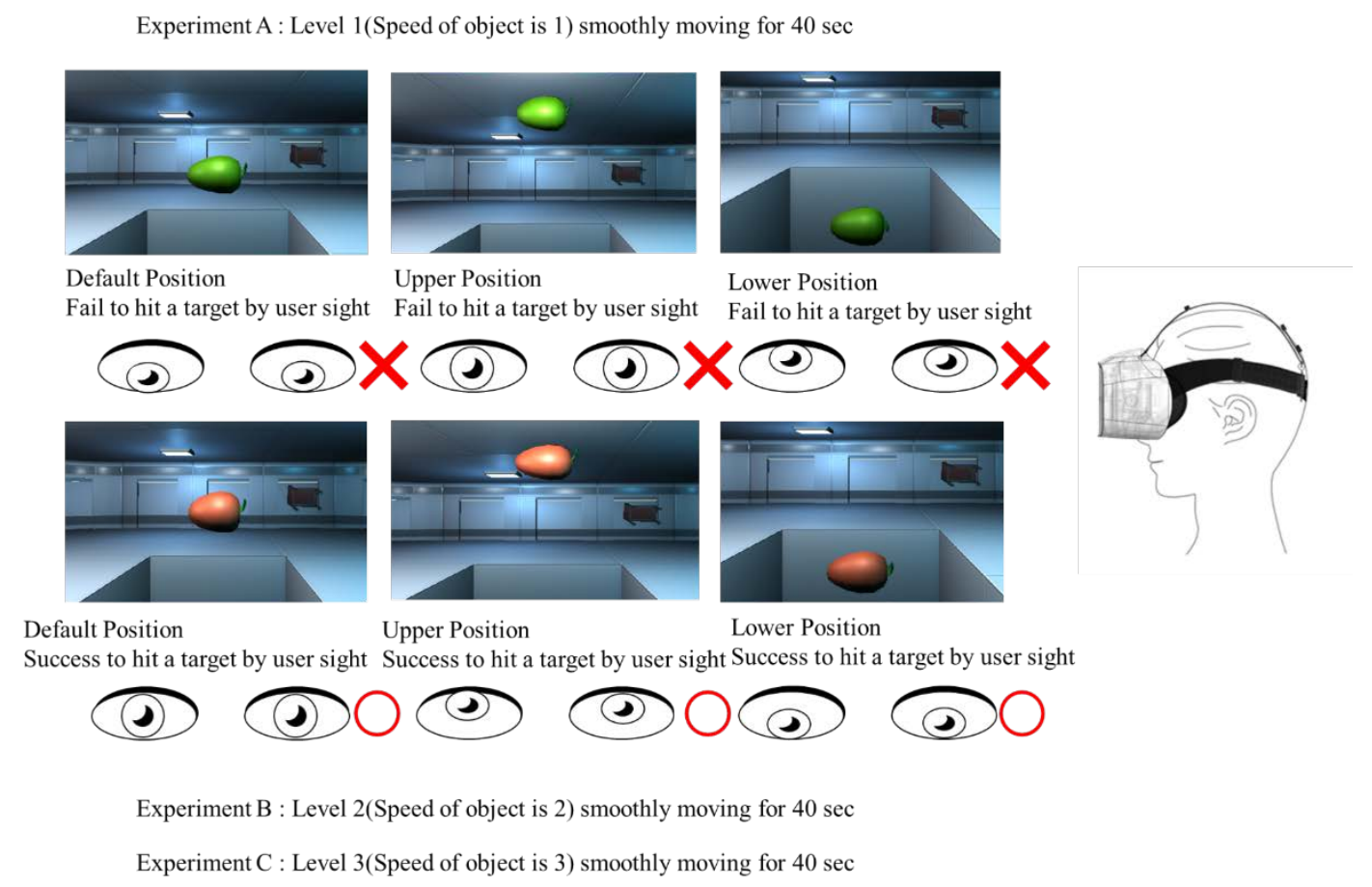

Fig. 6. The proposed vestibular rehabilitation content II for Cawthorne-Cooksey training

\subsubsection{Eye \& Head movement exercise: the proposed vestibular rehabilitation content III}

During the proposed vestibular rehabilitation content III, targets ("meteors") moved in random directions, and at random distances from the user. The user was required to follow the targets with their eyes and thus fire "bullets" at them, similar to a "first-person shooter" game. Fig. 7 shows the proposed vestibular rehabilitation content III for Herdman training with first-person shooter type.

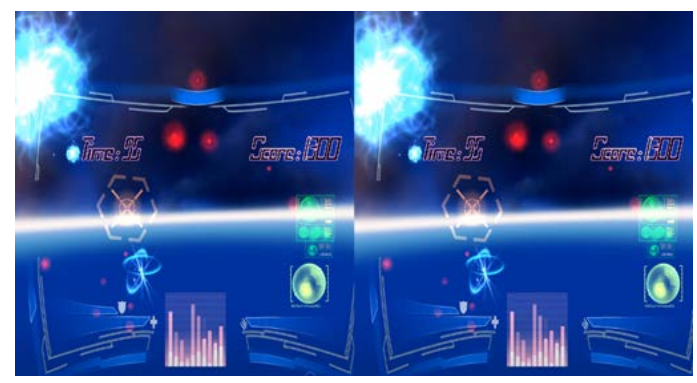

Fig. 7. The proposed vestibular rehabilitation content III for Herdman training 


\subsection{Statistical analysis}

Differences in scores (minimum, maximum, and average) between the healthy control and patient groups were analyzed for the three exercises, and scatter plots were generated to graph these differences. We recorded eye movements at 70 frames per second and omitted blink data. A p-value $<0.05$ was considered significant. The analyses were performed using SPSS for Windows software ([1.0.0.1298]; SPSS Inc., Chicago, IL, USA).

\section{Experimental Classification Results and Analysis}

The usability of the VR-based vestibular rehabilitation system was evaluated in 9 patients with unilateral vestibular dysfunction and 10 healthy subjects in a single testing session. All participants that satisfied the inclusion criteria received instructions in the VR-based vestibular rehabilitation exercises during a 20-min session and then completed a full 20-30 min training session. Table 2 shows the characteristics of the healthy controls and patients; the average age was $30.7 \pm 3.8$ and $52.0 \pm 15.3$, respectively. The gender ratios were $5: 5$ in the healthy control group and 4:5 in the patient group. Three patients had a diagnosis of benign paroxysmal positional vertigo, two had vascular necrosis, one had vascular necrosis, one had Meniere's disease, and two exhibited vestibular dysfunction. For healthy group the vestibular are not affected by age factor and both healthy control group and patient group are for comparison with score and accuracy during the test.

Table 2. Characteristics of participants

\begin{tabular}{ccc|cccc}
\hline \multicolumn{3}{c|}{ Healthy Subject } & \multicolumn{4}{c}{ Patient } \\
\hline Id & Sex & Age & Id & Sex & Age & Diagnosis \\
H1 & M & 32 & P1 & M & 67 & $\begin{array}{c}\text { Benign paroxysmal } \\
\text { position vertigo }\end{array}$ \\
H2 & M & 34 & P2 & M & 56 & Vestibular dysfunction \\
H3 & M & 31 & P3 & M & 36 & Meniere \\
H4 & M & 27 & P4 & M & 38 & Vascular necrosis \\
H5 & M & 29 & P5 & F & 66 & $\begin{array}{c}\text { Benign paroxysmal } \\
\text { position vertigo }\end{array}$ \\
H6 & F & 38 & P6 & F & 35 & Vascular necrosis \\
H7 & F & 34 & P7 & F & 77 & Vestibular dysfunction \\
H8 & F & 28 & P8 & F & 50 & Benign paroxysmal \\
& & & & & & position vertigo \\
H9 & F & 28 & P9 & F & 43 & Acute vestibular neuritis \\
H10 & F & 26 & & & & \\
\hline
\end{tabular}

Table 3 and Fig. 8(A) showed the average of game content I score in the healthy and patient groups. Depending on the difficulty of game content I, the healthy group showed $31.58 \pm 0.40$ in the level l, 29.24 \pm 0.84 in the level 2 and $28.66 \pm 0.60$ in the level 3 , and the patient group showed $21.85 \pm 2.66$ in the level $1,18.05 \pm 1.95$ in the level 2, 16.29 \pm 2.37 in the level 3, respectively. The healthy group scored almost 10 points higher than the patient group depending on the different level and these differences in content I were statistically significant ( $p=0.001, p=0.000$, and $p=0.000$ ). Additionally, Table 3 and Fig. 8(B) showed the eye-movement accuracy in the healthy and patient groups. Depending on the difficulty of game content I the healthy group showed $94.32 \pm 1.27$ in the level $1,88.27 \pm 2.36$ in the level 2 
and $90.06 \pm 1.90$ in the level 3 , and the patient group showed $65.99 \pm 8.04$ in the level 1 , $54.32 \pm 6.04$ in the level 2, 50.20 \pm 7.39 in the level 3, respectively. The eye-movement accuracy of healthy group in content I showed higher than the patient group depending on the different level and these differences were statistically significant ( $p=0.002, p=0.000$, and $p=0.000$ ).

Table 3. Average of game score and eye-movement accuracy in the proposed vestibular rehabilitation content I

\begin{tabular}{ccccccc}
\hline & \multicolumn{3}{c}{ Average of game score } & & \multicolumn{3}{c}{ Eye-movement accuracy (\%) } \\
\cline { 2 - 7 } & Level 1 & Level 2 & Level 3 & Level 1 & Level 2 & Level 3 \\
\hline $\begin{array}{c}\text { Healthy } \\
(\mathrm{n}=10)\end{array}$ & $31.58 \pm 0.40$ & $29.24 \pm 0.84$ & $28.66 \pm 0.60$ & $94.32 \pm 1.27$ & $88.27 \pm 2.36$ & $90.06 \pm 1.90$ \\
$\begin{array}{c}\text { Patient } \\
(\mathrm{n}=9)\end{array}$ & $21.85 \pm 2.66$ & $18.05 \pm 1.95$ & $16.29 \pm 2.37$ & $65.99 \pm 8.04$ & $54.32 \pm 6.04$ & $50.20 \pm 7.39$ \\
\hline$p$ & 0.001 & 0.000 & 0.000 & 0.002 & 0.000 & 0.000 \\
\hline
\end{tabular}

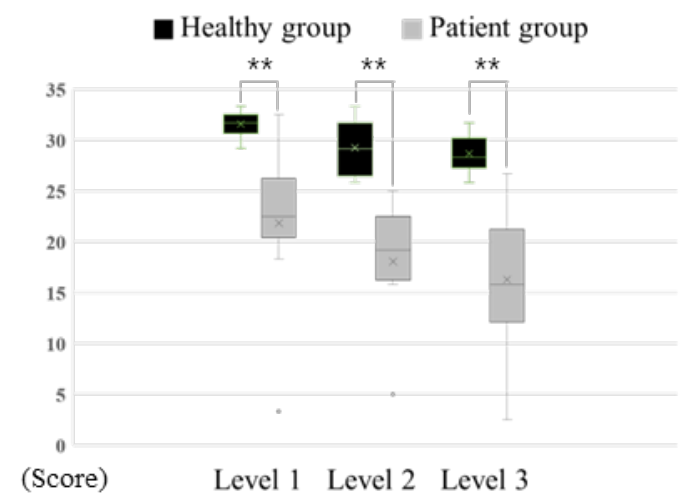

(a) game score

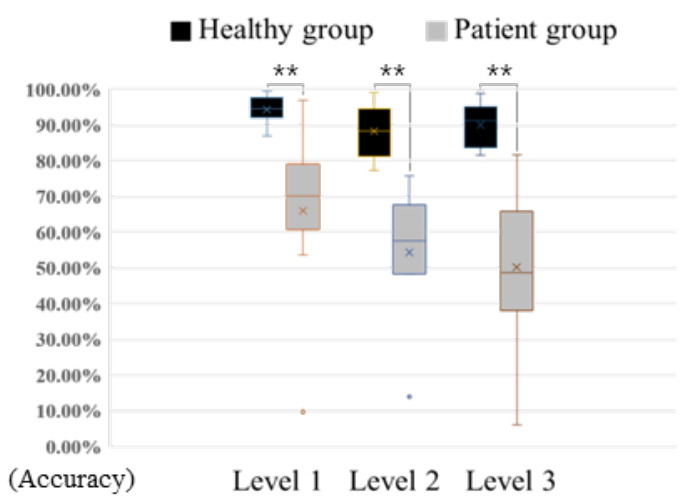

(b) eye-movement accuracy

Fig. 8. Comparison of game score and eye-movement accuracy in the proposed vestibular rehabilitation content I

Table 4 and Fig. 9(A) showed the average of game content II score in the healthy and patient groups. Depending on the difficulty of game content II the healthy group showed $30.83 \pm 0.45$ in the level $1,30.33 \pm 0.45$ in the level 2 and $29.58 \pm 0.82$ in the level 3 , and the patient group showed $23.70 \pm 1.25$ in the level $1,23.05 \pm 1.12$ in the level 2, 21.39 \pm 0.50 in the level 3, respectively. The healthy group scored about 8 points higher than the patient group depending on the different level and these differences in content II were statistically significant $(p=0.000$, $p=0.000$, and $p=0.000$ ). Additionally, Table 4 and Fig. 9(B) showed the eye-movement accuracy in the healthy and patient groups. Depending on the difficulty of game content I the healthy group showed $92.77 \pm 1.47$ in the level $1,91.77 \pm 1.49$ in the level 2 and $92.07 \pm 1.59$ in the level 3, and the patient group showed $72.27 \pm 3.75$ in the level 1, 69.31 \pm 3.63 in the level 2, $67.23 \pm 1.98$ in the level 3, respectively. The eye-movement accuracy of healthy group in content II showed higher than the patient group depending on the different level and these differences were statistically significant ( $p=0.002, p=0.000$, and $p=0.000$ ). 
Table 4. Average of game score and eye-movement accuracy in the proposed vestibular rehabilitation content II

\begin{tabular}{ccccccc}
\hline & \multicolumn{3}{c}{ Average of game score } & & \multicolumn{2}{c}{ Eye-movement accuracy (\%) } \\
\cline { 2 - 6 } & Level 1 & Level 2 & Level 3 & Level 1 & Level 2 & Level 3 \\
\hline $\begin{array}{c}\text { Healthy } \\
(\mathrm{n}=10)\end{array}$ & $30.83 \pm 0.45$ & $30.33 \pm 0.45$ & $29.58 \pm 0.82$ & $92.77 \pm 1.47$ & $91.77 \pm 1.49$ & $92.07 \pm 1.59$ \\
$\begin{array}{c}\text { Patient } \\
(\mathrm{n}=9)\end{array}$ & $23.70 \pm 1.25$ & $23.05 \pm 1.12$ & $21.39 \pm 0.50$ & $72.27 \pm 3.75$ & $69.31 \pm 3.63$ & $67.23 \pm 1.98$ \\
\hline$p$ & 0.000 & 0.000 & 0.000 & 0.002 & 0.000 & 0.000 \\
\hline
\end{tabular}

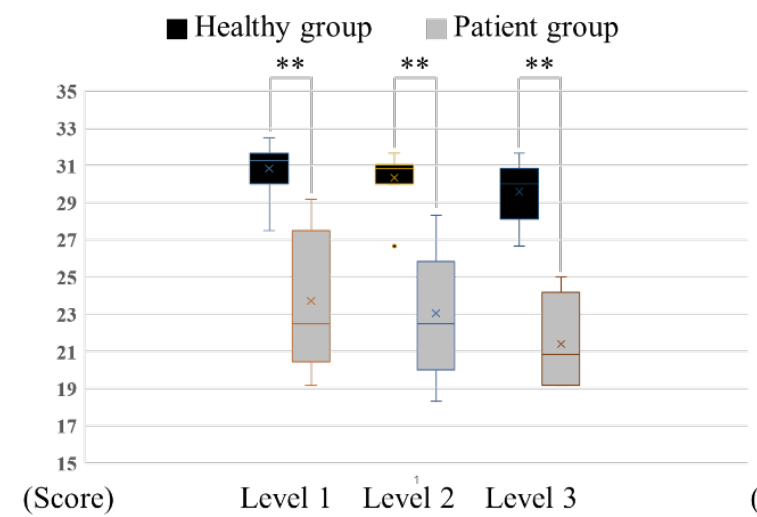

(a) game score

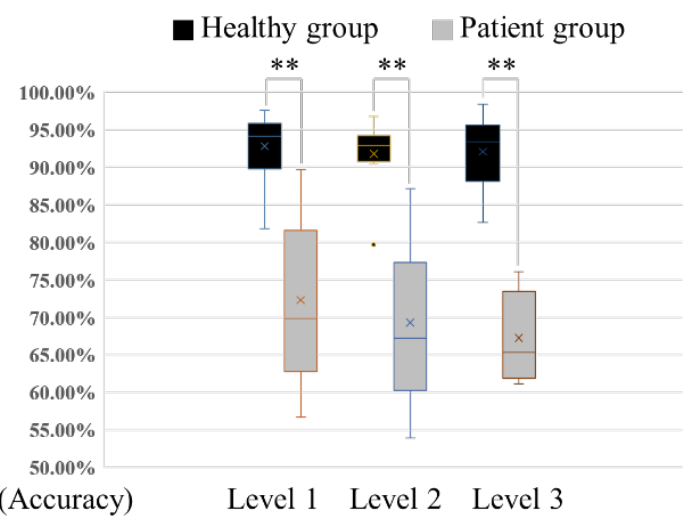

(b) eye-movement accuracy

Fig. 9. Comparison of the proposed vestibular rehabilitation content II in healthy and patient groups

Table 5 and Fig. 10 showed the average of game content III score in the healthy and patient groups. The healthy group showed $97.50 \pm 1.18$, and the patient group showed $79.11 \pm 7.30$ respectively. The healthy group scored 18 points higher than the patient group these differences were statistically significant $(\mathrm{p}=0.018)$.

Table 5. Average of game score for the proposed vestibular rehabilitation content III

\begin{tabular}{cc} 
& Content III \\
\hline $\begin{array}{c}\text { Healthy } \\
(\mathrm{n}=10)\end{array}$ & $97.50 \pm 1.18$ \\
$\begin{array}{c}\text { Patient } \\
(\mathrm{n}=9)\end{array}$ & $79.11 \pm 7.30$ \\
\hline$p$ & 0.018 \\
\hline
\end{tabular}




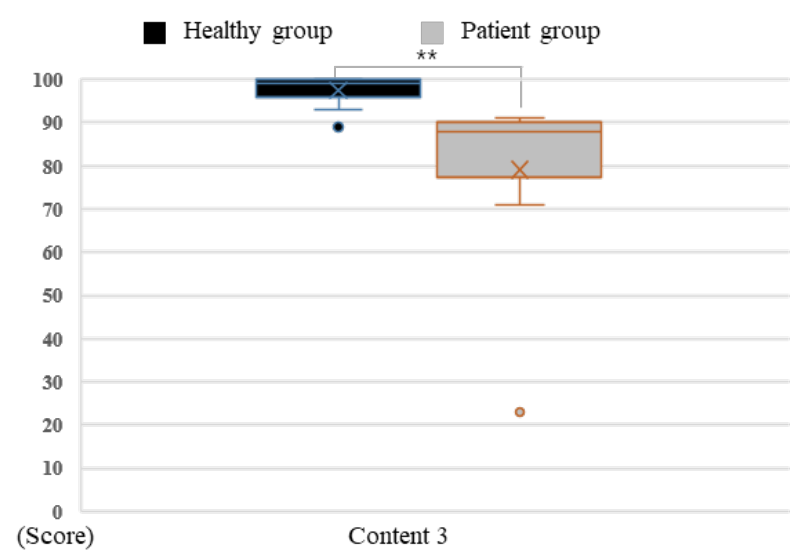

Fig. 10. Comparison of the proposed vestibular rehabilitation content III in healthy and patient groups

Fig. 11 showed eye-movement in the healthy and patient group. The eye-movement in healthy group was more concentrated than the patient group. The results implied that eye-movement in vestibular rehabilitation could be used as an indicator depending on the patient's condition or disease.

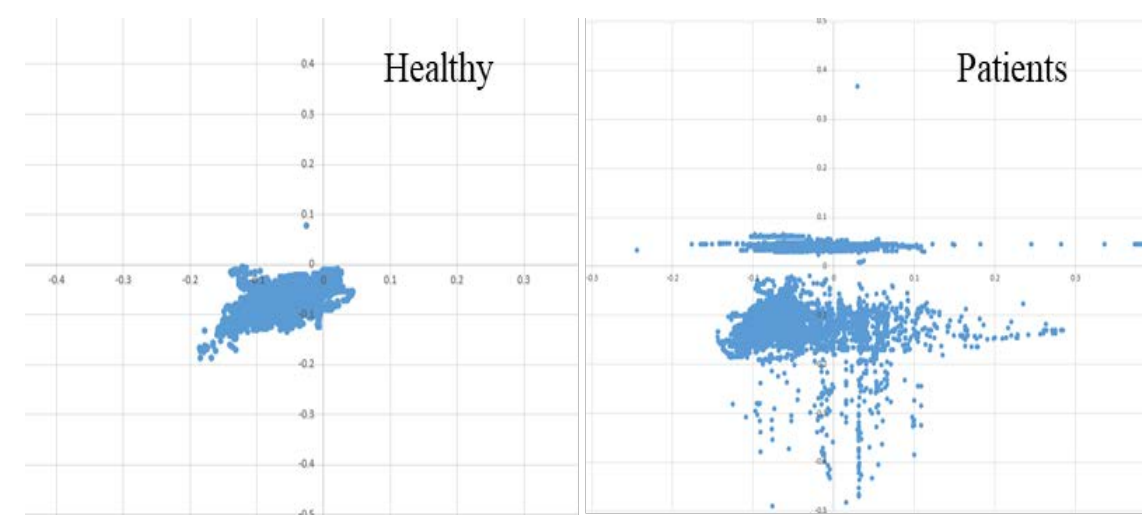

(a) Healty group

(b) Patients group

Fig. 11. Scatergram about eye-movement during the proposed vestibular rehabilitation content I and II

\section{Conclusion}

In this paper, VR-based vestibular rehabilitation exercises created using the Unity3D engine were tested with healthy controls and patients. A limitation of such rehabilitation training is that stimuli alerting the user to the need for concentration cannot be provided. Moreover, space and financial restrictions may limit applicability, and falls and collision can occur during training. However, VR-based rehabilitation training provides an interesting and immersive environment, which may improve patient interest, and remains much safer than conventional rehabilitation training. The use of an eye tracking-enabled HMD can increase data accuracy; HMDs without eye tracking capabilities can only detect head direction and degree of tilt, 
whereas newer models can track progress in real-time and provide feedback to the patient. As VR-based rehabilitation training becomes more realistic, we expect to further improve the rehabilitation exercises developed in this research. Furthermore, we plan to get more data from more people to make specific relation between our exercises and patient's condition of their symptom. In that case the data can have high quality such as accuracy and high reliability, that would be the first step of home training rehabilitation which prove that value.

\section{References}

[1] Neuhauser HK, von Brevern M, Radtke A, Lezius F, Feldmann M, Ziese T, Lempert T, "Epidemiology of vestibular vertigo: a neurotologic survey of the general population Neurology," 65(6), 898-904, 2005. Article (CrossRef Link)

[2] CRANE, Benjamin T., et al., Peripheral vestibular disorders. Otolaryngology: Head \& Neck Surgery, 5th ed, Mosby Elsevier, Pa, Philadelphia, 2010.

[3] D.E.Bamiou and L.M.Luxon, "Vertigo: clinical management and rehabilitation," Scott-Brown's Otorhinolaryngology, Head and Neck Surgery, M.GleesonandL. M.Luxon,Eds., CRCPress, NewYork, NY,USA, 7thedition, pp.3791-3817, 2008.

[4] R.E.Gans, "Vestibular rehabilitation: critical decision analysis," Seminars in Hearing, vol.23, no.2, pp.149-160, 2002. Article (CrossRef Link)

[5] Bergeron M, Lortie CL, Guitton MJ, "Use of virtual reality tools for vestibular disorders rehabilitation: a comprehensive analysis," Adv Med, vol. 2015, Article ID 916735, 2015. Article (CrossRef Link)

[6] Cohen HS, Gottshall KR, Graziano M, Malmstrom EM, Sharpe MH, "International survey of vestibular rehabilitation therapists by the Barany Society Ad Hoc Committee on Vestibular Rehabilitation Therapy,” J Vestib Res, 19(1-2), 15-20, 2009.

[7] Hecker HC, Haug CO, Herndon JW, "Treatment of the vertiginous patient using Cawthorne's vestibular exercises,” Laryngoscope, 84(11), 2065-2072, 1974. Article (CrossRef Link)

[8] Hillier SL, McDonnell M, "Vestibular rehabilitation for unilateral peripheral vestibular dysfunction," Clin Otolaryngol, 36(3), 248-249, 2011. Article (CrossRef Link)

[9] Whitney SL, Sparto PJ, Hodges LF, Babu SV, Furman JM, Redfern MS, "Responses to a virtual reality grocery store in persons with and without vestibular dysfunction," Cyberpsychol Behav., 9(2), 152-156, 2006. Article (CrossRef Link)

[10] Park JH, Jeon HJ, Lim EC, Koo JW, Lee HJ, Kim HJ, Lee JS, Song CG, Hong SK, "Feasibility of Eye Tracking Assisted Vestibular Rehabilitation Strategy Using Immersive Virtual Reality,” Clin Exp Otorhinolaryngol, 12(4), 376-384, 2019 May 9. Article (CrossRef Link)

[11] Monge Pereira E, Molina Rueda F, Alguacil Diego IM, Cano de la Cuerda R, de Mauro A, Miangolarra Page JC, "Use of virtual reality systems as proprioception method in cerebral palsy: clinical practice guideline,” Neurologia, 29(9), 550-559, 2011. Article (CrossRef Link)

[12] Meldrum D, Herdman S, Vance R, Murray D, Malone K, Duffy D, et al, "Effectiveness of conventional versus virtual reality-based balance exercises in vestibular rehabilitation for unilateral peripheral vestibular loss: results of a randomized controlled trial," Arch Phys Med Rehabil, 96(7), 1319-1328, 2015. Article (CrossRef Link)

[13] Pavlou M, Kanegaonkar RG, Swapp D, Bamiou DE, Slater M, Luxon LM, "The effect of virtual reality on visual vertigo symptoms in patients with peripheral vestibular dysfunction: a pilot study," $J$ Vestib Res, 22(5-6), 273-281, 2012.

[14] Alahmari KA, Sparto PJ, Marchetti GF, Redfern MS, Furman JM, Whitney SL, “Comparison of virtual reality based therapy with customized vestibular physical therapy for the treatment of vestibular disorders," IEEE Trans Neural Syst Rehabil Eng., 22(2), 389-399, 2014. Article (CrossRef Link)

[15] Yeh SC, Chen S, Wang PC, Su MC, Chang CH, Tsai PY, "Interactive 3-dimensional virtual reality rehabilitation for patients with chronic imbalance and vestibular dysfunction," Technol Health Care, 22(6), 915-21, 2014. Article (CrossRef Link) 
[16] Black FO, Pesznecker SC, “Vestibular adaptation and rehabilitation,” Curr Opin Otolaryngol Head Neck Surg. 11(5), 355-60, 2003. Article (CrossRef Link)

[17] Sparrer, Duong Dinh TA, Ilgner J, Westhofen M, "Vestibular rehabilitation using the Nintendo® Wii Balance Board -- a user-friendly alternative for central nervous compensation,” Acta Otolaryngol, 133(3), 239-245, 2013. Article (CrossRef Link)

[18] Meldrum D, Herdman S, Vance R, Murray D, Malone K, Duffy D, et al, "Effectiveness of conventional versus virtual reality-based balance exercises in vestibular rehabilitation for unilateral peripheral vestibular loss: results of a randomized controlled trial," Arch Phys Med Rehabil, 96(7), 1319-28, 2015. Article (CrossRef Link)

[19] T. Cawthorne, “The physiological basis for head exercises,” J Char Soc Physiother, Vol. 3, pp. 106-107, 1944.

[20] F. S. Cooksey, “Rehabilitation in vestibular injuries,” pp. 273-278, 1946.

[21] F. B. Horak, C. Jones-Rycewicz, F. O. Black and A. Shumway-Cook, "Effects of vestibular rehabilitation on dizziness and imbalance," Otolaryngology--head and neck surgery: official journal of American Academy of Otolaryngology-Head and Neck Surgery., Vol. 106, no. 2, pp. 175-180, 1992.

[22] SJ. Herdman, “Exercise strategies in vestibular disorders,” Ear Nose Throa.t, Vol. 68, No. 12, pp. 961-964, 1989.

[23] H. S. Cohen and K. T. Kimball, "Increased independence and decreased vertigo after vestibular rehabilitation,” Otolaryngology - Head and Neck Surgery., Vol. 128, No. 1, pp. 60-70, 2003. Article (CrossRef Link)

[24] Chung Ku Rhee, Jae Yun Jung, Yong Won Chung, "Practice of Vestibular Rehabilitation,” J Korean Balance Soc, Vol. 5, No. 1, pp. 149-157, 2006.

[25] Burdea Grigore, C., and P. Coiffet, "Virtual reality technology,” London: Wiley-Interscience, 1994.

[26] Changhoon Park, "Development of a Tangible Snowboard Training Simulator based on Virtual Reality,” Journal of Korea Game Society, 14(4), 87-94, Aug 2014. Article (CrossRef Link)

[27] Steuer, Jonathan, "Defining virtual reality: Dimensions determining telepresence," Journal of communication, 42(4), 73-93, 1992. Article (CrossRef Link)

[28] Jong-Gook Ko, Kyung-Nam Kim and R.S.Ramakrishna, "Facial Feature Tracking for Eye-Head Controlled Human Computer Interface," 1999.

[29] Creighton, Ryan Henson, "Unity 3D game development by example: A Seat-of-your-pants manual for building fun, groovy little games quickly,” Packt Publishing Ltd, 2010. 

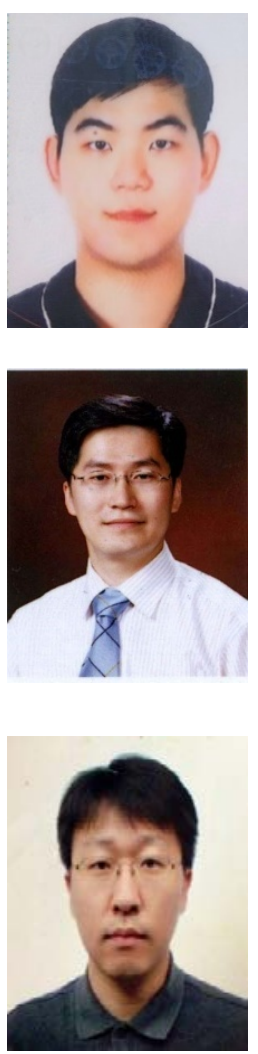

Sungyeup Kim is Chief Technology Officer of one of the leading ICT company, Bluecore Corporation. He received B.A. in Computer Science from Boston University in 1995. He also received MS in Information Systems from George Washington University in 1997. Now he is taking Ph.D degree of ICT Convergence Smart Rehabilitation from Soonchunhyang University. His research interests are A.I., Machine Learning, VR/AR.

E-mail: sykim@sch.ac.kr

Seong Jun Choi received his M.S from Chosun University in 2004, and Ph.D. received from Ajou University in 2013. Now he is professor of Otorhinolaryngology, Soonchunhyang University Cheonan Hospital. His research interests are Hearing loss, hearing rehabilitation and language rehabilitation.

E-mail: akas9238@hanmail.net 